
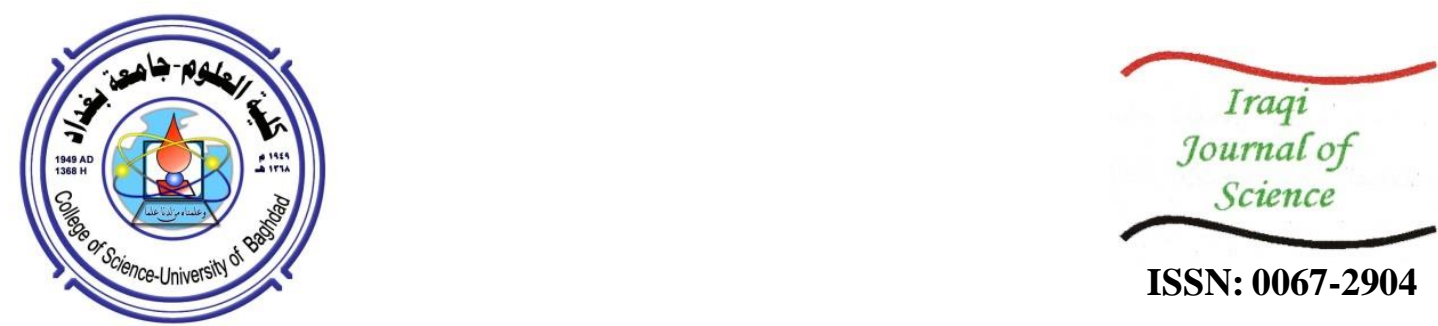

\title{
Solvability of Impulsive Nonlinear Partial Differential Equations with Nonlocal Conditions
}

\author{
Ali Kadhim Jabbar*, Sameer Qasim Hasan \\ Department of Mathematics, College of Education, Al-Mustansiriyah University, Baghdad, Iraq \\ Received: 8/12/ 2019 \\ Accepted: $15 / 3 / 2020$

\begin{abstract}
The aim of this paper is to investigate the theoretical approach for solvability of impulsive abstract Cauchy problem for impulsive nonlinear fractional order partial differential equations with nonlocal conditions, where the nonlinear extensible beam equation is a particular application case of this problem.
\end{abstract}

Keywords: fractional calculus, semigroup theorems, fixed point theorem, nonlocal conditions and mild solution.

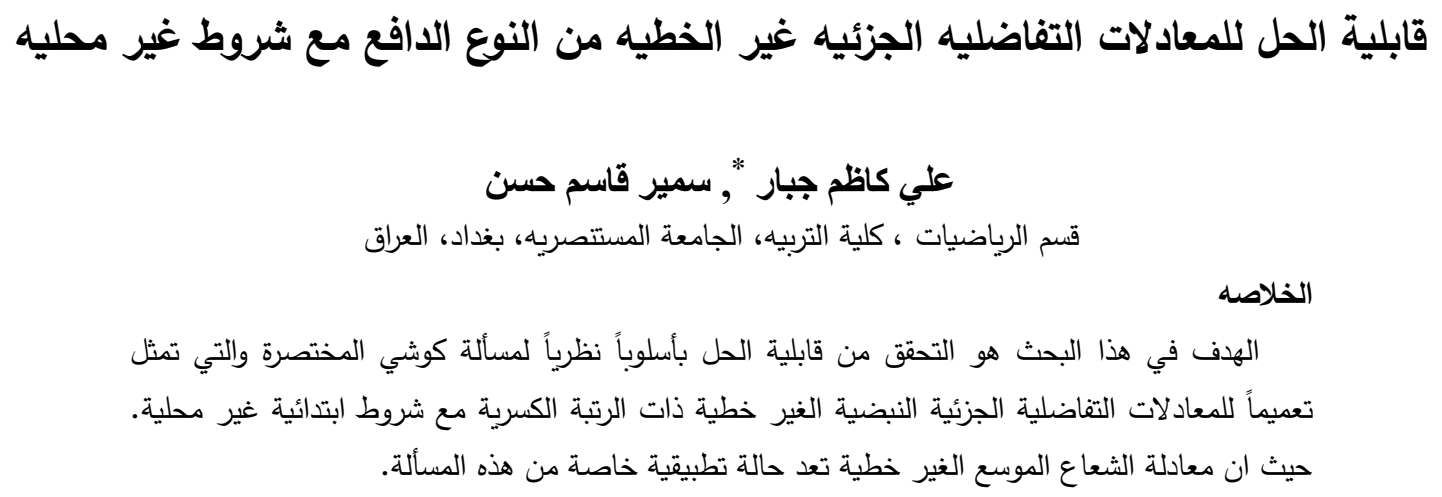

\section{Introduction}

The fractional differential equations are models of many applications, such as medicine, engineering, physics and other sciences. They are becoming more important tools among researchers and have been attracting many authors in recent years $[1,2,3,4,5,6,7,8,9,10]$. Furthermore, fractional impulsive differential equations played a main role in the modeling phenomena, for example in describing population dynamics which are subject to abrupt changes, as well as other phenomena such as diseases, harvesting, and so forth. From this reason, there are many researchers who discussed the existence of a mild solution of impulsive fractional differential equations $[11,12,13,14,15,16$, 17]. There are some authors who also studied impulsive fractional differential equations with delay $[18,19,20]$. However, there are few authors who discussed the existence of mild solutions of the impulsive fractional integro-differential equations of order $1<\alpha \leq 2$.

In this work, we will study the existence of mild solutions for considered an abstract Cauchy problem, which is entitled the impulsive nonlinear fractional order partial differential equations with nonlocal conditions, as follows: 


$$
\begin{aligned}
& \begin{array}{c}
\left({ }^{C} D_{t}^{\alpha}\left[z(t, x)-h\left(t, z_{t}(t, x)\right)\right]+{ }^{C} D_{x}^{2 \alpha} z(t, x)-\left(\beta_{1}+\beta_{2} \int_{0}^{L}\left|\frac{\partial z(y, s)}{\partial y}\right|^{2} d y\right){ }^{C} D_{x}^{\alpha} z(t, x)\right. \\
=\int_{0}^{t} f\left(s, z_{s}(s, x), I_{s}^{\gamma} z\right) d s,
\end{array} \\
& \text { for } t \in J=[0, T], \quad t \neq t_{k}, \quad k=1,2, \ldots . m ., \quad 1<\alpha, \gamma \leq 2 \text {, } \\
& \left\{\left.\Delta z(t, x)\right|_{t=t_{k}}=I_{k}\left(z\left(t_{k}^{-}\right)\right)\right. \text {, } \\
& \left.\Delta z^{\prime}(t, x)\right|_{t=t_{k}}=\bar{I}_{k}\left(z\left(t_{k}^{-}\right)\right) \text {, } \\
& z_{0}(t, x)+g(t, z)=\varphi(t) \in E_{v}, \quad t \in(-\infty, 0], \\
& z^{\prime}(0, x)=z_{1} \in \bar{E}_{v} \text {. }
\end{aligned}
$$

Now, consider the following $A^{\alpha} z(t, x)={ }^{C} D_{x}^{\alpha} z(t, x)$. We have

$$
\left\{\begin{array}{l}
{ }^{C} D_{t}^{\alpha}\left[z(t, x)-h\left(t, z_{t}(t, x)\right)\right]=\hat{A}^{\alpha} z(t, x)+\int_{0}^{t} f\left(s, z_{s}(s, x), I_{s}^{\gamma} z\right) d s, \\
\text { for } t \in J=[0, T], \quad t \neq t_{k}, \quad k=1.2 \ldots . m, \quad 1<\alpha, \gamma \leq 2, \\
\left.\Delta z(t, x)\right|_{t=t_{k}}=I_{k}\left(z\left(t_{k}^{-}\right)\right), \\
\left.z^{\prime}(t, x)\right|_{t=t_{k}}=\bar{I}_{k}\left(z\left(t_{k}^{-}\right)\right), \\
z(t, x)+g(t, z)=\varphi(t) \in E_{v}, \\
z^{\prime}(0, x)=z_{1}, \quad h^{\prime}\left(0, z_{t}(0, x)\right)=z_{2} .
\end{array}\right.
$$

where $\hat{A}^{\alpha}=\left(\beta_{1}+\beta_{2} M\left[\left\|A^{\alpha / 2} z\right\|^{2}\right]\right) A^{\alpha}-A^{2 \alpha}$ is a linear operator in the Banach space $X,{ }^{C} D^{\alpha}$, $I^{\gamma}$ are the Caputo fractional derivative and fractional integration of $1<\alpha, \gamma \leq 2$, respectively, $z(t, x)$ belongs to Banach space $X$, and $\left.\Delta z(t, x)\right|_{t=t_{k}}$ is defined by $\left.\Delta z(t, x)\right|_{t=t_{k}}=z\left(t_{k}^{+}\right)-z\left(t_{k}^{-}\right)$. Also, $\left.\Delta z^{\prime}(t, x)\right|_{t=t_{k}}$ is defined by $\left.\Delta z^{\prime}(t, x)\right|_{t=t_{k}}=z^{\prime}\left(t_{k}^{+}\right)-z^{\prime}\left(t_{k}^{-}\right)$for all $k=1,2 \ldots, m, 0<t_{0}<t_{1}<$ $t_{2}<\cdots<t_{m}<t_{m+1}=T$, with $z\left(t_{k}^{+}\right), z\left(t_{k}^{-}\right)$representing the right and left limits of $z$ at $t_{k}$ respectively, $z^{\prime}\left(t_{k}^{+}\right), z^{\prime}\left(t_{k}^{-}\right)$representing the right and left limits of $z^{\prime}$ at $t_{k}$ respectively, and $g: E_{v} \rightarrow X$ is a given function. Let $z_{t}($.$) denoted by z_{t}(\theta)=z(t+\theta), \theta \in(-\infty, 0]$.

Many scientific problem of the impulsive nonlinear fractional order partial differential equations with nonlocal conditions can be expressed as in the above systems (1.1) and (1.2). Some of them do not have easy solvability. They are even difficult to study sometimes. Also their behaviors for their solutions is not appearing in general and, thus, we need more effort and practices. Therefore, these problems have taken our interest.

The aim of this article is to study and present all the results of the solvability for the proposal class impulsive nonlinear fractional order partial differential equations with nonlocal conditions. Some of special types of cases of the proposed problem have been approached, using nonlinear functional analysis theorems and abstract Cauchy problem involving semigroup operators with Krasnoselskii's fixed point theorem, to show the solvability with some recent sufficient and necessary conditions.

\section{Preliminaries}

In this section, we present some assumptions, notation and results needed in our proofs later.

\section{Assumptions (2.1)}

The following Assumptions of $E_{v}$ are needed in the description of piecewise continuous space $P C((-\infty, 0], X)$.

1. The continuous function $v:(-\infty, 0] \rightarrow(0,+\infty)$ is satisfying $l=\int_{-\infty}^{0} v(t) d t<+\infty$ :, and $\left(E_{v},\|\cdot\|_{E_{v}}\right)$ by a Banach space induced by the function $v$ is defined as follows:

$E_{v}=\{\omega:(-\infty, 0] \rightarrow X$ : for any $c>0, \omega(\theta)$ is a bounded and measurable function on

$$
\left.[-c, 0] \text {, and } \int_{-\infty}^{0} v(s) \sup _{s \leq \theta \leq 0}\|\omega(\theta)\| d s<+\infty\right\}
$$

with the norm $\|\omega\|_{E_{v}}=\int_{-\infty}^{0} v(s) \sup _{s \leq \theta \leq 0}\|\omega(\theta)\| d s$.

2. Let the space $\bar{E}_{v}=\left\{\omega, \omega^{\prime}:(-\infty, T] \rightarrow X ; \omega_{k}, \omega_{k}^{\prime} \in C\left(J_{k}, X\right), k=0,1,2, \ldots, m\right.$ and there exist $\omega\left(t_{k}^{+}\right), \omega\left(t_{k}^{-}\right)$and $\omega^{\prime}\left(t_{k}^{+}\right), \omega^{\prime}\left(t_{k}^{-}\right)$with $\omega\left(t_{k}\right)=\omega\left(t_{k}^{-}\right)$and $\omega^{\prime}\left(t_{k}\right)=\omega^{\prime}\left(t_{k}^{-}\right), \omega_{0}=\omega(0)+$ $\left.g(\omega)=\omega \in E_{v}\right\}$ where $\omega_{k}, \omega_{k}{ }^{\prime}$ are the restrictions of $\omega, \omega^{\prime}$ to $J_{k}$, where $J_{0}=\left[0, t_{1}\right], J_{k}=\left(t_{k}, t_{k+1}\right]$, $k=0,1,2, \ldots, m$. Define the seminorm in the space $\bar{E}_{v}$ by $\|\omega\|_{\bar{E}_{v}}=\|\omega\|_{E_{v}}+\sup \{\|\omega(s)\|: s \in[0, T]\}$ for $\omega \in \bar{E}_{v}$ and $\left\|\omega^{\prime}\right\|_{\bar{E}_{v}}=\sup \left\{\left\|\omega^{\prime}(s)\right\|: s \in[0, T]\right\}$ for $\omega^{\prime} \in \bar{E}_{v}$. 
3. By the space $\bar{E}_{v}$ we can define the space $\left(\overline{\bar{E}}_{v},\|\cdot\|_{\bar{E}_{v}}\right)$ as:

$\overline{\bar{E}}_{v}=\left\{\omega \in \bar{E}_{v}: 0=\omega_{0} \in E_{v}\right\}$ with norm $\|\omega\|_{\bar{E}_{v}}=\sup \{\|\omega(s)\|: s \in[0, T]\}$.

4. Let the space $E_{r}=\left\{\omega \in \bar{E}_{v}:\|\varphi\|_{E_{v^{\prime}}} \leq r\right\}$ for $r>0$, then $E_{r}$ for each $r$, is a closed convex, bounded subset in $X$.

Definition (2.1), [21]

The fractional integral of order $\alpha>0$ with the lower limit 0 for a function $\mathrm{h}$ is defined as:

$I^{\alpha} h(t)=\frac{1}{\Gamma(\alpha)} \int_{0}^{t} \frac{h(s)}{(t-s)^{1-\alpha}} d s, t>0, \alpha>0$

where $\Gamma(\mathrm{u})=\int_{0}^{\infty} \mathrm{e}^{-\mathrm{s}} \mathrm{s}^{\mathrm{u}-1} \mathrm{ds}, u>0$ (gamma function).

Definition (2.2), [21]

The Caputo fractional derivative of order $\alpha>0$ with lower limit 0 for a function $\mathrm{h}$ can be written as:

${ }^{C} D^{\alpha} h(t)=\frac{1}{\Gamma(n-\alpha)} \int_{0}^{t} \frac{h^{(n)}(s)}{(t-s)^{\alpha+1-n}} d s, t>0, n-1<\alpha \leq n$.

Definition (2.3), [21]

The Laplace transform, of the Caputo fractional derivative of order $\alpha>0$ is given as:

$\mathcal{L}\left\{{ }^{C} D_{t}^{\alpha} h(t)\right\}(\lambda)=\lambda^{\alpha} \mathcal{L}(h)(\lambda)-\left.\sum_{k=0}^{n-1} \lambda^{\alpha-k-1}\left[D^{k} h(t)\right]\right|_{t=0}, \quad n-1<\alpha \leq n$.

Lemma (2.1), [22]

Let $\in E_{v^{\prime}}$, for $t \in J$, then $z_{t} \in E_{v}$ and $l\|z(t)\| \leq\left\|z_{t}\right\|_{E_{v}} \leq\|\varphi\|_{E_{v}}+l \sup _{0 \leq s \leq t}\|z(s)\|$.

Definition (2.4), [10]

Let $A: D \subseteq X \rightarrow X$ be a closed linear operator. Then $A$ is the sectorial operator of type $(M, \theta, \alpha, \mu)$ if there exist $0<\theta<\frac{\pi}{2}, M>0$ and $\mu \in \mathbb{R}$ such that the $\alpha$-resolvent of $A$ exists outside the secto

$\mu+S_{\theta}=\left\{\mu+\lambda^{\alpha}: \lambda \in \mathbb{C},\left|\operatorname{Arg}\left(-\lambda^{\alpha}\right)\right|<\theta\right\}$

and $\quad\left\|\left(\lambda^{\alpha} I-A\right)^{-1}\right\| \leq \frac{M}{\left|\lambda^{\alpha}-\mu\right|}, \lambda^{\alpha} \notin \mu+S_{\theta}$.

Lemma (2.2), [10]

Let $M \neq \varnothing$ be a closed convex subset of a Banach space $X$. Let $Y$ and $\Psi$ be two operators which satisfy

1. $Y u+\Psi v \in M$ whenever $u, v \in M$;

2. $\Psi$ is continuous and compact;

3. $Y$ is a contraction.

Then there exists $z \in M$ such that $z=\Upsilon_{Z}+\Psi_{z}$.

Lemma (2.3), [23]

Let $A$ be a densely defined operator in $X$ satisfying the following conditions ;

(i) For some $0<\theta<\frac{\pi}{2}, \mu+S_{\theta}=\{\mu+\lambda: \lambda \in \mathbb{C},|\operatorname{Arg}(-\lambda)|<\theta\}$.

(ii) There exists a constant $M$ such that

$$
\left\|(\lambda I-A)^{-1}\right\| \leq \frac{M}{|\lambda-\mu|}, \lambda \notin \mu+S_{\theta} .
$$

Then, $A$ is the infinitesimal generator of a semigroup $T(t)$ satisfying $\|T(t)\| \leq C$. Moreover,

$T(t)=\frac{1}{2 \pi i} \int_{c} e^{\lambda t} R(\lambda, A) d \lambda$ with $c$ being a suitable path $\lambda \notin \mu+S_{\theta}$ for $\lambda \in c$.

Lemma (2.4)

If $\hat{A}^{\alpha}$ is a densely defined sectorial operator of type $(M, \theta, \alpha, \mu)$, then $\hat{A}^{\alpha}$ is the infinitesimal generator of a $\alpha$-resolvent family $\left\{T_{\alpha}(t)\right\}_{t \geq 0}$ in Banach space $X$, where

$T_{\alpha}(t)=\frac{1}{2 \pi i} \int_{c} e^{\lambda t} R\left(\lambda^{\alpha}, \hat{A}^{\alpha}\right) d \lambda$.

Proof

Since $\hat{A}^{\alpha}$ is sectorial operator then, form definition (2.4), we get $\hat{A}^{\alpha}$ is closed linear operator and satisfies

(i) For some $0<\theta<\frac{\pi}{2}, \mu+S_{\theta}=\left\{\mu+\lambda^{\alpha}: \lambda \in \mathbb{C},\left|\operatorname{Arg}\left(-\lambda^{\alpha}\right)\right|<\theta\right\}$.

(ii) There exists a constant $M$ such that

$$
\left\|\left(\lambda^{\alpha} I-A\right)^{-1}\right\| \leq \frac{M}{\left|\lambda^{\alpha}-\mu\right|}, \lambda^{\alpha} \notin \mu+S_{\theta} .
$$


Also, $\hat{A}^{\alpha}$ is densely defined operator. By lemma (2.3), then $\hat{A}^{\alpha}$ is the infinitesimal generator of a $\alpha$ resolvent family $\left\{T_{\alpha}(t)\right\}_{t \geq 0}$ in Banach space, where

Lemma (2.5), [10]

$$
T_{\alpha}(t)=\frac{1}{2 \pi i} \int_{c} e^{\lambda t} R\left(\lambda^{\alpha}, \hat{A}^{\alpha}\right) d \lambda .
$$

If the sectorial operator $\hat{A}^{\alpha}$ is of type $(M, \theta, \alpha, \mu)$ in definition (2.4), then we have the following:

(i) If $\mu \geq 0$ and $\phi \in(0, \pi)$, then we have that

$$
\begin{aligned}
& \left\|S_{\alpha}(t)\right\| \leq \frac{K_{1}(\theta, \phi) M e^{\left[K_{1}(\theta, \phi)\left(1+\mu t^{\alpha}\right)\right]^{\frac{1}{\alpha}}\left[\left(1+\frac{\sin \phi}{\sin (\phi-\theta)}\right)^{\frac{1}{\alpha}}-1\right]}}{\pi \sin ^{1+\frac{1}{\alpha}} \theta}\left(1+\mu t^{\alpha}\right) \\
& +\frac{\Gamma(\alpha) M}{\pi\left(1+\mu t^{\alpha}\right)\left|\cos \frac{\pi-\phi}{\alpha}\right|^{\alpha} \sin \theta \sin \phi} \\
& \left\|T_{\alpha}(t)\right\| \leq \frac{M\left[\left(1+\frac{\sin \phi}{\sin (\phi-\theta)}\right)^{\frac{1}{\alpha}}-1\right]}{\pi \sin \theta}\left(1+\mu t^{\alpha}\right)^{\frac{1}{\alpha} t^{\alpha-1}} e^{\left[K_{1}(\theta, \phi)\left(1+\mu t^{\alpha}\right)\right]^{\frac{1}{\alpha}}} \\
& +\frac{M t^{\alpha-1}}{\pi\left(1+\mu t^{\alpha}\right)\left|\cos \frac{\pi-\phi}{\alpha}\right|^{\alpha} \sin \theta \sin \phi} \\
& \left\|K_{\alpha}(t)\right\| \leq \frac{M\left[\left(1+\frac{\sin \phi}{\sin (\phi-\theta)}\right)^{\frac{1}{\alpha}}-1\right] K_{1}(\theta, \phi)}{\pi \sin \theta^{\frac{\alpha+2}{\alpha}}}\left(1+\mu t^{\alpha}\right)^{\frac{\alpha-1}{\alpha}} t^{\alpha-1} e^{\left[K_{1}(\theta, \phi)\left(1+\mu t^{\alpha}\right)\right]^{\frac{1}{\alpha}}} \\
& +\frac{M \alpha \Gamma(\alpha)}{\pi\left(1+\mu t^{\alpha}\right)\left|\cos \frac{\pi-\phi}{\alpha}\right|^{\alpha} \sin \theta \sin \phi}
\end{aligned}
$$

for $t>0$, where $K_{1}(\theta, \phi)=\max \left\{1, \frac{\sin \phi}{\sin (\phi-\theta)}\right\}$.

(ii) If $\mu<0$ and $\phi \in(0, \pi)$, then we have that

$$
\begin{aligned}
& \left\|S_{\alpha}(t)\right\| \leq\left(\frac{e M\left[(1+\sin \phi)^{\frac{1}{\alpha}}-1\right]}{\pi|\cos \phi|^{1+\frac{1}{\alpha}}}+\frac{\Gamma(\alpha) M}{\pi|\cos \phi|\left|\cos \frac{\pi-\phi}{\alpha}\right|^{\alpha}}\right) \frac{1}{1+|\mu| t^{\alpha}} \\
& \left\|T_{\alpha}(t)\right\| \leq\left(\frac{e M\left[(1+\sin \phi)^{\frac{1}{\alpha}}-1\right]}{\pi|\cos \phi|}+\frac{M}{\pi|\cos \phi|\left|\cos \frac{\pi-\phi}{\alpha}\right|}\right) \frac{t^{\alpha-1}}{1+|\mu| t^{\alpha}} \\
& \left\|K_{\alpha}(t)\right\| \leq\left(\frac{e M\left[(1+\sin \phi)^{\frac{1}{\alpha}}-1\right] t}{\pi|\cos \phi|^{1+\frac{2}{\alpha}}}+\frac{\alpha \Gamma(\alpha) M}{\pi|\cos \phi|\left|\cos \frac{\pi-\phi}{\alpha}\right|}\right) \frac{1}{1+|\mu| t^{\alpha}}, \quad \text { for } t>0 .
\end{aligned}
$$

\section{Main results}

To investigate the existence of the mild solution of the impulsive abstract Cauchy problem (1.2), we assume the following conditions:

$\left(\mathrm{A}_{1}\right)$ The semigroups $S_{\alpha}(t), K_{\alpha}(t), P_{\alpha}(t)$ and $T_{\alpha}(t)$ generated by the operator $\hat{A}^{\alpha}$ are compact in $\overline{D\left(\hat{A}^{\alpha}\right)}$ when $t \geq 0$ and

$\sup _{t \in J}\left\|S_{\alpha}(t)\right\| \leq \breve{M}, \sup _{t \in J}\left\|K_{\alpha}(t)\right\| \leq \breve{M}, \sup _{t \in J}\left\|P_{\alpha}(t)\right\| \leq \breve{M}, \sup _{t \in J}\left\|T_{\alpha}(t)\right\| \leq \breve{M}$.

$\left(\mathrm{A}_{2}\right)$ The $h: J \times E_{v} \rightarrow X$ and there exist constants $L_{1}, L_{2}>0$ satisfying

$\left\|h\left(t_{1}, \varphi_{1}\right)-h\left(t_{2}, \varphi_{2}\right)\right\| \leq L_{1}\left(\left\|\varphi_{1}-\varphi_{2}\right\|_{E_{v}}+\left\|t_{1}-t_{2}\right\|\right)$

$\sup _{t \in J}\|h(t, 0)\|=L_{2}, h^{\prime}\left(0, z_{t}(0, x)\right)=z_{2}$.

$\left(\mathrm{A}_{3}\right)$ The $f: J \times E_{v} \times X \rightarrow X$ and there exist constants $K_{1}, K_{2}>0$ satisfying

$\left\|f\left(t_{1}, \varphi_{1}, y_{1}\right)-f\left(t_{2}, \varphi_{2}, y_{2}\right)\right\| \leq K_{1}\left(\left\|\varphi_{1}-\varphi_{2}\right\|_{E_{v}}+\left\|y_{1}-y_{2}\right\|+\left\|t_{1}-t_{2}\right\|\right)$

$\sup _{t \in J}\|f(t, 0,0)\|=K_{2}$.

$\left(\mathrm{A}_{4}\right)$ The $I_{k}: X \rightarrow X, \bar{I}_{k}: X \rightarrow X$ and there exist constants $a_{k}, b_{k}>0$ and $c_{k}, d_{k}>0$,

$k=1,2, \ldots, m$ satisfying

$\left\|I_{k}\left(z_{1}\right)-I_{k}\left(z_{2}\right)\right\| \leq a_{k}\left\|z_{1}-z_{2}\right\|, \quad\left\|I_{k}(0)\right\| \leq b_{k}$,

$\left\|\left(\hat{A}^{\alpha}\right)^{-1} \bar{I}_{k}\left(z_{1}\right)-\left(\hat{A}^{\alpha}\right)^{-1} \bar{I}_{k}\left(z_{2}\right)\right\| \leq c_{k}\left\|z_{1}-z_{2}\right\|, \quad\left\|\left(\hat{A}^{\alpha}\right)^{-1} \bar{I}_{k}(0)\right\| \leq d_{k}$.

$\left(\mathrm{A}_{5}\right)$ The $g: J \times E_{v} \rightarrow X$ and there exists a constant $N_{1}>0$ satisfying 


$$
\left\|g\left(t_{1}, \varphi_{1}\right)-g\left(t_{2}, \varphi_{2}\right)\right\| \leq N_{1}\left(\left\|\varphi_{1}-\varphi_{2}\right\|_{E_{v}}+\left\|t_{1}-t_{2}\right\|\right)
$$

$\left(\mathrm{A}_{6}\right)$ Let

$$
\begin{aligned}
\rho_{1}= & \breve{M}[\|\varphi(0)\|+\|g(0, u+\emptyset)\|+\|h(0, \varphi(0)-g(0, u+\emptyset))\|]+\breve{M}\left\|z_{1}-z_{2}\right\| \\
& +\breve{M} t_{1}\left(L_{1} r^{\prime}+L_{2}\right)+\breve{M} t_{1} T\left(K_{1}\left(r^{\prime}+\frac{t^{\gamma}}{\Gamma(\gamma+1)} r^{\prime}\right)+K_{2}\right), \\
\rho_{2}= & \sum_{i=1}^{k} 2 \breve{M}\left(\breve{M}\|\varphi(0)-g(0, u+\emptyset)-h(0, \varphi(0)-g(0, u+\emptyset))\|+\breve{M}\left\|z_{1}-z_{2}\right\|\right) \\
& \quad+\sum_{i=1}^{k} \breve{M}\left(a_{i}\left(r^{\prime \prime}\right)+b_{i}-\left\|z_{1}-z_{2}\right\|\right) \\
+ & \sum_{i=1}^{k} \bar{M}\left(c_{i}\left(r^{\prime \prime}\right)+d_{i}+\|\varphi(0)-g(0, u+\emptyset)-h(0, \varphi(0)-g(0, u+\emptyset))\|\right) \\
& +\sum_{i=1}^{k} 2 \breve{M}\left(\left(t_{i}-t_{i-1}\right) \breve{M}\left(L_{1}\left(r^{\prime}\right)+L_{2}\right)+\left(t_{i}-t_{i-1}\right) \breve{M} T\left(K_{1}\left(r^{\prime}+\frac{t^{\gamma}}{\Gamma(\gamma+1)}\left(r^{\prime}\right)\right)+K_{2}\right)\right. \\
+ & \breve{M} T\left(L_{1}\left(r^{\prime}\right)+L_{2}\right)+\breve{M} T^{2}\left(K_{1}\left(r^{\prime}+\frac{t^{\gamma}}{\Gamma(\gamma+1)}\left(r^{\prime \prime}\right)\right)+K_{2}\right) \text { for } t \in\left(t_{k}, t_{k+1}\right] .
\end{aligned}
$$

where $r^{\prime}=\|\varphi(0)\|_{E_{v}}+l r+\breve{M}\|[\varphi(0)-g(0, u+\emptyset)]\|$ and $r^{\prime \prime}=r+\breve{M}\|[\varphi(0)-g(0, u+\varnothing)]\|$ then we can get $\rho_{1}, \rho_{2}>0$, such that

$$
\mathrm{N} \leq\left\{\begin{array}{l}
\rho_{1}, t \in\left[0, t_{1}\right] \\
\rho_{2}, t \in\left[t_{k}, t_{k+1}\right], k=1,2, \ldots, m
\end{array} \leq r .\right.
$$

(A $\mathrm{A}_{7}$ 1) $\bar{M}\left(1+L_{1}\right) N_{1}<1$,

2) $\left[\sum_{i=1}^{k} 2 \breve{M} N_{1}\left(\breve{M}\left(1+L_{1}\right)\right)+\sum_{i=1}^{k} \breve{M} d_{i}+\sum_{i=1}^{k} \breve{M}\left(\bar{d}_{i}+N_{1}+L_{1}\left(N_{1}\right)\right)\right]<1$.

\section{Lemma (3.6)}

If the sectorial operator $\hat{A}^{\alpha}$ of type $(M, \theta, \alpha, \mu)$ and $f: J \times E_{v} \times X \rightarrow X$ is a map that satisfies $\left(\mathrm{A}_{3}\right)$, then the impulsive abstract Cauchy problem (1.2) is equivalent to the integral equation given by the following:

$$
\begin{aligned}
& \quad \text { if } t \in(-\infty, 0] . \\
& =\left\{\begin{array}{cc}
\varphi(t), & \text { if } t \in\left(0, t_{1}\right] \\
\quad S_{\alpha}(t)[\varphi(0)-g(0, z)-h(0, \varphi(0)-g(0, z))]+K_{\alpha}(t)\left[z_{1}-z_{2}\right] & \\
\quad+\int_{0}^{t} P_{\alpha}(t-s) h\left(s, z_{s}(s, x)\right) d s & \quad+\int_{0}^{t} \int_{0}^{s} T_{\alpha}(t-s) f\left(c, z_{c}(c, x), I_{c}^{\gamma} z\right) d c d s, \\
& \sum_{i=1}^{k}\left[S_{\alpha}\left(t-t_{i}\right)+K_{\alpha}\left(t-t_{i}\right)\right]\left(S_{\alpha}\left(t_{i}\right)[\varphi(0)-g(0, z)-h(0, \varphi(0)-g(0, z))]\right. \\
& +K_{\alpha}\left(t_{i}\right)\left[z_{1}-z_{2}\right]+\int_{t_{i-1}}^{t_{i}} P_{\alpha}\left(t_{i}-s\right) h\left(s, z_{s}(s, x)\right) d s \\
+ & \left.\int_{t_{i-1}}^{t_{i}} \int_{0}^{s} T_{\alpha}\left(t_{i}-s\right) f\left(c, z_{c}(c, x), I_{c}^{\gamma} z\right) d c d s\right)+\sum_{i=1}^{k} S_{\alpha}\left(t-t_{i}\right)\left(I_{i}\left(z\left(t_{i}^{-}\right)\right)-\left[z_{1}-z_{2}\right]\right) \\
& +\sum_{i=1}^{k} K_{\alpha}\left(t-t_{i}\right)\left(\left(\hat{A}^{\alpha}\right)^{-1} \bar{I}_{i}\left(z\left(t_{i}^{-}\right)\right)-[\varphi(0)-g(0, z)-h(0, \varphi(0)-g(0, z))]\right) \\
& +\int_{t_{k}}^{t} P_{\alpha}(t-s) h\left(s, z_{s}(s, x)\right) d s+\int_{t_{k}}^{t} \int_{0}^{s} T_{\alpha}(t-s) f\left(c, z_{c}(c, x), I_{c}^{\gamma} z\right) d c d s, \\
& \text { if } t \in\left(t_{k}, t_{k+1}\right], \quad k=1,2, \ldots, m
\end{array}\right.
\end{aligned}
$$

where, $S_{\alpha}(t)=\frac{1}{2 \pi i} \int_{0}^{\infty} e^{\lambda t} \lambda^{\alpha-1}\left(\lambda^{\alpha} I-\hat{A}^{\alpha}\right)^{-1} d \lambda$

$$
\begin{aligned}
& K_{\alpha}(t)=\frac{1}{2 \pi i} \int_{0}^{\infty} e^{\lambda t} \lambda^{\alpha-2}\left(\lambda^{\alpha} I-\hat{A}^{\alpha}\right)^{-1} d \lambda \\
& P_{\alpha}(t)=\frac{1}{2 \pi i} \int_{0}^{\infty} e^{\lambda t} \lambda^{\alpha}\left(\lambda^{\alpha} I-\hat{A}^{\alpha}\right)^{-1} d \lambda \\
& T_{\alpha}(t)=\frac{1}{2 \pi i} \int_{0}^{\infty} e^{\lambda t}\left(\lambda^{\alpha} I-\hat{A}^{\alpha}\right)^{-1} d \lambda
\end{aligned}
$$

Such that $\lambda^{\alpha} \notin \mu+S_{\theta}$ for $\lambda \in \mathbb{C}$.

\section{Proof}

By integrating the both sides of impulsive abstract Cauchy problem (1.2) of order $1<\alpha \leq 2$, we get the equivalent equation

$$
\begin{aligned}
z(t, x)= & {[\varphi(0)-g(0, z)-h(0, \varphi(0)-g(0, z))]+\left[z_{1}-z_{2}\right] t+h\left(t, z_{t}(t, x)\right) } \\
& +\frac{1}{\Gamma(\alpha)} \int_{0}^{t}(t-s)^{\alpha-1} \hat{A}^{\alpha} z(s, x) d s+\frac{1}{\Gamma(\alpha)} \int_{0}^{t} \int_{0}^{s}(t-s)^{\alpha-1} f\left(c, z_{c}(c, x), I_{c}^{\gamma} z\right) d c d s
\end{aligned}
$$

Now, by taking a Laplace transform of both sides of (3.3), we have

$$
\mathcal{L}\{z(t, x)\}(\lambda)=\frac{1}{\lambda}[\varphi(0)-g(0, z)-h(0, \varphi(0)-g(0, z))]+\frac{1}{\lambda^{2}}\left[z_{1}-z_{2}\right]+\mathcal{L}\left\{h\left(t, z_{t}(t, x)\right)\right\}(\lambda)
$$




$$
\begin{aligned}
&+\frac{1}{\lambda^{\alpha}} \hat{A}^{\alpha} \mathcal{L}\{z(t, x)\}(\lambda)+\frac{1}{\lambda^{\alpha}} \mathcal{L}\left\{\int_{0}^{s} f\left(c, z_{c}(c, x), I_{c}^{\gamma} z\right) d c\right\}(\lambda) \\
& \mathcal{L}\{z(t, x)\}(\lambda)= \lambda^{\alpha-1}\left(\lambda^{\alpha} I-\hat{A}^{\alpha}\right)^{-1}[\varphi(0)-g(z)-h(0, \varphi(0)-g(z))] \\
&+\lambda^{\alpha-2}\left(\lambda^{\alpha} I-\hat{A}^{\alpha}\right)^{-1}\left[z_{1}-z_{2}\right] \\
&+\lambda^{\alpha}\left(\lambda^{\alpha} I-\hat{A}^{\alpha}\right)^{-1} \mathcal{L}\left\{h\left(t, z_{t}(t, x)\right)\right\}(\lambda)+\left(\lambda^{\alpha} I-\hat{A}^{\alpha}\right)^{-1} \mathcal{L}\left\{\int_{0}^{s} f\left(c, z_{c}(c, x), I_{c}^{\gamma} z\right) d c\right\}(\lambda)(3.2)
\end{aligned}
$$

Now, by taking inverse transform of Laplace, we get

$$
\begin{aligned}
& z(t, x)=\frac{1}{2 \pi i} \int_{0}^{\infty} e^{\lambda t} \lambda^{\alpha-1}\left(\lambda^{\alpha} I-\hat{A}^{\alpha}\right)^{-1}[\varphi(0)-g(0, z)-h(0, \varphi(0)-g(0, z))] d \lambda \\
& +\frac{1}{2 \pi i} \int_{0}^{\infty} e^{\lambda t} \lambda^{\alpha-2}\left(\lambda^{\alpha} I-\hat{A}^{\alpha}\right)^{-1}\left[z_{1}-z_{2}\right] d \lambda+\frac{1}{2 \pi i} \int_{0}^{\infty} e^{\lambda t} \lambda^{\alpha}\left(\lambda^{\alpha} I-\hat{A}^{\alpha}\right)^{-1} \mathcal{L}\left\{h\left(t, z_{t}(t, x)\right)\right\} d \lambda \\
& \quad+\frac{1}{2 \pi i} \int_{0}^{\infty} e^{\lambda t}\left(\lambda^{\alpha} I-\hat{A}^{\alpha}\right)^{-1} \mathcal{L}\left\{\int_{0}^{s} f\left(c, z_{c}(c, x), I_{c}^{\gamma} z\right) d c\right\} d \lambda
\end{aligned}
$$

By Laplace transform of the last term of (3.4), we have

$$
\begin{aligned}
z(t, x) & =\frac{1}{2 \pi i} \int_{0}^{\infty} e^{\lambda t} \lambda^{\alpha-1}\left(\lambda^{\alpha} I-\hat{A}^{\alpha}\right)^{-1}[\varphi(0)-g(0, z)-h(0, \varphi(0)-g(0, z))] d \lambda \\
& +\frac{1}{2 \pi i} \int_{0}^{\infty} e^{\lambda t} \lambda^{\alpha-2}\left(\lambda^{\alpha} I-\hat{A}^{\alpha}\right)^{-1}\left[z_{1}-z_{2}\right] d \lambda \\
& +\frac{1}{2 \pi i} \int_{0}^{\infty} \int_{0}^{t} e^{\lambda(t-s)} \lambda^{\alpha}\left(\lambda^{\alpha} I-\hat{A}^{\alpha}\right)^{-1} h\left(s, z_{s}(s, x)\right) d s d \lambda \\
& +\frac{1}{2 \pi i} \int_{0}^{\infty} \int_{0}^{t} \int_{0}^{s} e^{\lambda(t-s)}\left(\lambda^{\alpha} I-\hat{A}^{\alpha}\right)^{-1} f\left(c, z_{c}(c, x), I_{c}^{\gamma} z\right) d c d s d \lambda
\end{aligned}
$$

We can rewrite the equation (3.5) as follows:

$$
\begin{aligned}
z(t, x) & =S_{\alpha}(t)[\varphi(0)-g(0, z)-h(0, \varphi(0)-g(0, z))]+K_{\alpha}(t)\left[z_{1}-z_{2}\right] \\
& +\int_{0}^{t} P_{\alpha}(t-s) h\left(s, z_{s}(s, x)\right) d s+\int_{0}^{t} \int_{0}^{s} T_{\alpha}(t-s) f\left(c, z_{c}(c, x), I_{c}^{\gamma} z\right) d c d s
\end{aligned}
$$

where

$$
\begin{aligned}
& S_{\alpha}(t)=\frac{1}{2 \pi i} \int_{0}^{\infty} e^{\lambda t} \lambda^{\alpha-1}\left(\lambda^{\alpha} I-\hat{A}^{\alpha}\right)^{-1} d \lambda \\
& K_{\alpha}(t)=\frac{1}{2 \pi i} \int_{0}^{\infty} e^{\lambda t} \lambda^{\alpha-2}\left(\lambda^{\alpha} I-\hat{A}^{\alpha}\right)^{-1} d \lambda \\
& P_{\alpha}(t)=\frac{1}{2 \pi i} \int_{0}^{\infty} e^{\lambda t} \lambda^{\alpha}\left(\lambda^{\alpha} I-\hat{A}^{\alpha}\right)^{-1} d \lambda \\
& T_{\alpha}(t)=\frac{1}{2 \pi i} \int_{0}^{\infty} e^{\lambda t}\left(\lambda^{\alpha} I-\hat{A}^{\alpha}\right)^{-1} d \lambda
\end{aligned}
$$

If $t \in\left(t_{1}, t_{2}\right]$, then

$$
\begin{aligned}
& z(t, x)=S_{\alpha}\left(t-t_{1}\right)[\varphi(0)-g(0, z)-h(0, \varphi(0)-g(0, z))]+K_{\alpha}\left(t-t_{1}\right)\left[z_{1}-z_{2}\right] \\
& +\int_{t_{1}}^{t} P_{\alpha}(t-s) h\left(s, z_{s}(s, x)\right) d s+\int_{t_{1}}^{t} \int_{0}^{s} T_{\alpha}(t-s) f\left(c, z_{c}(c, x), I_{c}^{\gamma} z\right) d c d s \\
& \left.\Delta z(t, x)\right|_{t=t_{1}}=z\left(t_{1}^{+}\right)-z\left(t_{1}^{-}\right)=I_{1}\left(z\left(t_{1}^{-}\right)\right) \\
& =[\varphi(0)-g(0, z)-h(0, \varphi(0)-g(0, z))]+\left[z_{1}-z_{2}\right] \\
& -\left(S_{\alpha}\left(t_{1}\right)[\varphi(0)-g(0, z)-h(0, \varphi(0)-g(0, z))]\right. \\
& +K_{\alpha}\left(t_{1}\right)\left[z_{1}-z_{2}\right]+\int_{0}^{t_{1}} P_{\alpha}\left(t_{1}-s\right) h\left(s, z_{s}(s, x)\right) d s \\
& \left.+\int_{0}^{t_{1}} \int_{0}^{s} T_{\alpha}\left(t_{1}-s\right) f\left(c, z_{c}(c, x), I_{c}^{\gamma} z\right) d c d s\right)=I_{1}\left(z\left(t_{1}^{-}\right)\right) \\
& {[\varphi(0)-g(0, z)-h(0, \varphi(0)-g(0, z))]=-\left[z_{1}-z_{2}\right]} \\
& +\left(S_{\alpha}\left(t_{1}\right)[\varphi(0)-g(0, z)-h(0, \varphi(0)-g(0, z))]\right. \\
& +K_{\alpha}\left(t_{1}\right)\left[z_{1}-z_{2}\right]+\int_{0}^{t_{1}} P_{\alpha}\left(t_{1}-s\right) h\left(s, z_{s}(s, x)\right) d s \\
& \left.+\int_{0}^{t_{1}} \int_{0}^{s} T_{\alpha}\left(t_{1}-s\right) f\left(c, z_{c}(c, x), I_{c}^{\gamma} z\right) d c d s\right)+I_{1}\left(z\left(t_{1}^{-}\right)\right) \\
& \left.\Delta z^{\prime}(t, x)\right|_{t=t_{1}}=z^{\prime}\left(t_{1}^{+}\right)-z^{\prime}\left(t_{1}^{-}\right)=\bar{I}_{1}\left(z\left(t_{1}^{-}\right)\right) \\
& z^{\prime}\left(t_{1}^{+}\right)-z^{\prime}\left(t_{1}^{-}\right)=\hat{A}^{\alpha}[\varphi(0)-g(0, z)-h(0, \varphi(0)-g(0, z))]+\hat{A}^{\alpha}\left[z_{1}-z_{2}\right] \\
& -\left(\hat{A}^{\alpha} S_{\alpha}\left(t_{1}\right)[\varphi(0)-g(0, z)-h(0, \varphi(0)-g(0, z))]\right. \\
& +\hat{A}^{\alpha} K_{\alpha}\left(t_{1}\right)\left[z_{1}-z_{2}\right]+\int_{0}^{t_{1}} \hat{A}^{\alpha} P_{\alpha}\left(t_{1}-s\right) h\left(s, z_{s}(s, x)\right) d s \\
& \left.+\int_{0}^{t_{1}} \int_{0}^{s} \hat{A}^{\alpha} T_{\alpha}\left(t_{1}-s\right) f\left(c, z_{c}(c, x), I_{c}^{\gamma} z\right) d c d s\right)=\bar{I}_{1}\left(z\left(t_{1}^{-}\right)\right) \\
& {\left[z_{1}-z_{2}\right]=-[\varphi(0)-g(0, z)-h(0, \varphi(0)-g(0, z))]} \\
& +\left(S_{\alpha}\left(t_{1}\right)[\varphi(0)-g(0, z)-h(0, \varphi(0)-g(0, z))]\right.
\end{aligned}
$$




$$
\begin{aligned}
& +K_{\alpha}\left(t_{1}\right)\left[z_{1}-z_{2}\right]+\int_{0}^{t_{1}} P_{\alpha}\left(t_{1}-s\right) h\left(s, z_{s}(s, x)\right) d s \\
& \left.+\int_{0}^{t_{1}} \int_{0}^{s} T_{\alpha}\left(t_{1}-s\right) f\left(c, z_{c}(c, x), I_{c}^{\gamma} z\right) d c d s\right)+\left(\hat{A}^{\alpha}\right)^{-1} \bar{I}_{1}\left(z\left(t_{1}^{-}\right)\right)
\end{aligned}
$$

By (3.6), (3.7) and (3.8), we get that

$$
\begin{aligned}
z(t, x)=[ & \left.S_{\alpha}\left(t-t_{1}\right)+K_{\alpha}\left(t-t_{1}\right)\right]\left(S_{\alpha}\left(t_{1}\right)[\varphi(0)-g(0, z)-h(0, \varphi(0)-g(0, z))]\right. \\
& +K_{\alpha}\left(t_{1}\right)\left[z_{1}-z_{2}\right]+\int_{0}^{t_{1}} P_{\alpha}\left(t_{1}-s\right) h\left(s, z_{s}(s, x)\right) d s \\
& \left.+\int_{0}^{t_{1}} \int_{0}^{s} T_{\alpha}\left(t_{1}-s\right) f\left(c, z_{c}(c, x), I_{c}^{\gamma} z\right) d c d s\right) \\
& \quad S_{\alpha}\left(t-t_{1}\right)\left[z_{1}-z_{2}\right]+S_{\alpha}\left(t-t_{1}\right) I_{1}\left(z\left(t_{1}^{-}\right)\right) \\
- & K_{\alpha}\left(t-t_{1}\right)[\varphi(0)-g(0, z)-h(0, \varphi(0)-g(0, z))]+K_{\alpha}\left(t-t_{1}\right)\left(\hat{A}^{\alpha}\right)^{-1} \bar{I}_{1}\left(z\left(t_{1}^{-}\right)\right) \\
& +\int_{t_{1}}^{t} P_{\alpha}(t-s) h\left(s, z_{s}(s, x)\right) d s+\int_{t_{1}}^{t} \int_{0}^{s} T_{\alpha}(t-s) f\left(c, z_{c}(c, x), I_{c}^{\gamma} z\right) d c d s
\end{aligned}
$$

for $t \in\left(t_{1}, t_{2}\right]$, if $t \in\left(t_{k}, t_{k+1}\right]$, then we get

$$
\begin{aligned}
& z(t, x) \\
& =\left\{\begin{array}{cc}
\varphi(t), & \text { if } t \in(-\infty, 0] . \\
\quad S_{\alpha}(t)[\varphi(0)-g(0, z)-h(0, \varphi(0)-g(0, z))]+K_{\alpha}(t)\left[z_{1}-z_{2}\right] & \text { if } t \in\left(0, t_{1}\right] \\
& +\int_{0}^{t} P_{\alpha}(t-s) h\left(s, z_{s}(s, x)\right) d s \\
& +\int_{0}^{t} \int_{0}^{s} T_{\alpha}(t-s) f\left(c, z_{c}(c, x), I_{c}^{\gamma} z\right) d c d s, \\
& \sum_{i=1}^{k}\left[S_{\alpha}\left(t-t_{i}\right)+K_{\alpha}\left(t-t_{i}\right)\right]\left(S_{\alpha}\left(t_{i}\right)[\varphi(0)-g(0, z)-h(0, \varphi(0)-g(0, z))]\right. \\
& +K_{\alpha}\left(t_{i}\right)\left[z_{1}-z_{2}\right]+\int_{t_{i-1}}^{t_{i}} P_{\alpha}\left(t_{i}-s\right) h\left(s, z_{s}(s, x)\right) d s \\
+ & \left.\int_{t_{i-1}}^{t_{i}} \int_{0}^{s} T_{\alpha}\left(t_{i}-s\right) f\left(c, z_{c}(c, x), I_{c}^{\gamma} z\right) d c d s\right)+\sum_{i=1}^{k} S_{\alpha}\left(t-t_{i}\right)\left(I_{i}\left(z\left(t_{i}^{-}\right)\right)-\left[z_{1}-z_{2}\right]\right) \\
& +\sum_{i=1}^{k} K_{\alpha}\left(t-t_{i}\right)\left(\left(\hat{A}^{\alpha}\right)^{-1} \bar{I}_{i}\left(z\left(t_{i}^{-}\right)\right)-[\varphi(0)-g(0, z)-h(0, \varphi(0)-g(0, z))]\right) \\
& +\int_{t_{k}}^{t} P_{\alpha}(t-s) h\left(s, z_{s}(s, x)\right) d s+\int_{t_{k}}^{t} \int_{0}^{s} T_{\alpha}(t-s) f\left(c, z_{c}(c, x), I_{c}^{\gamma} z\right) d c d s, \\
& \text { if } t \in\left(t_{k}, t_{k+1}\right], \quad k=1,2, \ldots, m .
\end{array}\right.
\end{aligned}
$$

Now, we define the mild solution of impulsive abstract Cauchy problem (1.2) for every $z \in E_{r}$.

\section{Definition (3.5)}

A function $z:(-\infty, T] \rightarrow X$ is called a mild solution of the impulsive abstract Cauchy problem (1.2), if $z(t, x)+g(t, z)=\varphi(t) \in E_{v}$, the impulsive conditions $\left.\Delta z(t, x)\right|_{t=t_{k}}=I_{k}\left(z\left(t_{k}^{-}\right)\right),\left.\Delta z^{\prime}(t, x)\right|_{t=t_{k}}=$ $\bar{I}_{k}\left(z\left(t_{k}^{-}\right)\right) \quad k=1,2 \ldots, m$ are verified, the restriction of $z(\cdot)$ to the interval $J_{k}=\left[t_{k}, t_{k+1}\right]$ is continuous and the following integral equation holds for $t \in J$.

$z(t, x)$

$$
=\left\{\begin{array}{cc}
\varphi(t), & \text { if } t \in(-\infty, 0] . \\
\quad S_{\alpha}(t)[\varphi(0)-g(0, z)-h(0, \varphi(0)-g(0, z))]+K_{\alpha}(t)\left[z_{1}-z_{2}\right] & \text { if } t \in\left(0, t_{1}\right] . \\
& +\int_{0}^{t} P_{\alpha}(t-s) h\left(s, z_{s}(s, x)\right) d s \\
& +\int_{0}^{t} \int_{0}^{s} T_{\alpha}(t-s) f\left(c, z_{c}(c, x), I_{c}^{\gamma} z\right) d c d s, \\
& \sum_{i=1}^{k}\left[S_{\alpha}\left(t-t_{i}\right)+K_{\alpha}\left(t-t_{i}\right)\right]\left(S_{\alpha}\left(t_{i}\right)[\varphi(0)-g(0, z)-h(0, \varphi(0)-g(0, z))]\right. \\
& +K_{\alpha}\left(t_{i}\right)\left[z_{1}-z_{2}\right]+\int_{t_{i-1}}^{t_{i}} P_{\alpha}\left(t_{i}-s\right) h\left(s, z_{s}(s, x)\right) d s \\
+ & \left.\int_{t_{i-1}}^{t_{i}} \int_{0}^{s} T_{\alpha}\left(t_{i}-s\right) f\left(c, z_{c}(c, x), I_{c}^{\gamma} z\right) d c d s\right)+\sum_{i=1}^{k} S_{\alpha}\left(t-t_{i}\right)\left(I_{i}\left(z\left(t_{i}^{-}\right)\right)-\left[z_{1}-z_{2}\right]\right) \\
& +\sum_{i=1}^{k} K_{\alpha}\left(t-t_{i}\right)\left(\left(\hat{A}^{\alpha}\right)^{-1} \bar{I}_{i}\left(z\left(t_{i}^{-}\right)\right)-[\varphi(0)-g(0, z)-h(0, \varphi(0)-g(0, z))]\right) \\
& +\int_{t_{k}}^{t} P_{\alpha}(t-s) h\left(s, z_{s}(s, x)\right) d s+\int_{t_{k}}^{t} \int_{0}^{s} T_{\alpha}(t-s) f\left(c, z_{c}(c, x), I_{c}^{\gamma} z\right) d c d s, \\
& \text { if } t \in\left(t_{k}, t_{k+1}\right], \quad k=1,2, \ldots, m .
\end{array}\right.
$$




\section{Definition (3.6):}

Let $z(t, x)=y(t, x)+\emptyset(t, x)$ and for $\in E_{v}$. To introduce the following main theorem, we define the function $\varnothing$ as follows:

\section{Lemma (3.7)}

$$
\emptyset(t, x)=\left\{\begin{array}{l}
\varphi(t, x) \text { for } t \in(-\infty, 0], \\
S_{\alpha}(t)[\varphi(0)-g(z)] \text { for } t \in J .
\end{array}\right.
$$

If the assumptions $\left(\mathrm{A}_{1}\right)-\left(\mathrm{A}_{3}\right)$ hold, then the $\overline{\mathrm{Y}}\left(E_{r}\right)$ is equicontinuous, where the operator $\overline{\mathrm{Y}}$ on $E_{r}$ defined by

$$
(\overline{\mathrm{Y}} y)(t)=\left\{\begin{array}{c}
\int_{0}^{0}, \text { if } t \in(-\infty, 0] \\
\quad \begin{array}{c}
\left.\sum_{i=1}^{k} P_{\alpha}(t-s) h\left(s, y_{s}+\emptyset_{s}\right) d s+\int_{0}^{t} \int_{0}^{s} T_{\alpha}\left(t-t_{i}\right)+K_{\alpha}\left(t-t_{i}\right)\right]\left(\int_{t_{i-1}}^{t_{i}} P_{\alpha}\left(t_{i}-s\right) h\left(s, y_{s}+\emptyset_{s}\right) d s\right. \\
\left.\quad+\int_{t_{i-1}}^{t_{i}} \int_{0}^{s} T_{\alpha}\left(t_{i}-s\right) f\left(c, y_{c}+\emptyset_{c}, I_{c}^{\gamma}(y+\emptyset)\right) d c d s\right)+\int_{t_{k}}^{t} P_{\alpha}(t-s) h\left(s, y_{s}+\emptyset_{s}\right) d s \\
\quad+\int_{t_{k}}^{t} \int_{0}^{s} T_{\alpha}(t-s) f\left(c, y_{c}+\emptyset_{c}, I_{c}^{\gamma}(y+\emptyset)\right) d c d s, \quad \text { if } t \in\left(t_{k}, t_{k+1}\right], k=1,2, \ldots, m
\end{array}
\end{array}\right.
$$

\section{Proof}

For $y \in E_{r}$, if $s_{1}, s_{2} \in J$ and $0<s_{1}<s_{2} \leq t_{1}$, then we have

$$
\begin{aligned}
& \left\|(\overline{\mathrm{Y}} y)\left(s_{2}\right)-(\overline{\mathrm{Y}} y)\left(s_{1}\right)\right\| \\
& \leq \int_{0}^{s_{1}}\left\|P_{\alpha}\left(s_{2}-s\right)-P_{\alpha}\left(s_{1}-s\right)\right\|\left\|h\left(s, y_{s}+\emptyset_{s}\right)\right\| d s+\int_{s_{1}}^{s_{2}}\left\|P_{\alpha}\left(s_{1}-s\right)\right\|\left\|h\left(s, y_{s}+\emptyset_{s}\right)\right\| d s \\
& +\int_{0}^{s_{1}} \int_{0}^{s}\left\|T_{\alpha}\left(s_{2}-s\right)-T_{\alpha}\left(s_{1}-s\right)\right\|\left\|f\left(c, y_{c}+\emptyset_{c}, I_{c}^{\gamma}(y+\emptyset)\right)\right\| d c d s \\
& +\int_{s_{1}}^{s_{2}} \int_{0}^{s}\left\|T_{\alpha}\left(s_{1}-s\right)\right\|\left\|f\left(c, y_{c}+\emptyset_{c}, I_{c}^{\gamma}(y+\emptyset)\right)\right\| d c d s \\
& \leq s_{1}\left\|P_{\alpha}\left(s_{2}-s\right)-P_{\alpha}\left(s_{1}-s\right)\right\|\left(L_{1}\left(r^{\prime}\right)+L_{2}\right)+\left(s_{2}-s_{1}\right) \breve{M}\left(L_{1}\left(r^{\prime}\right)+L_{2}\right) \\
& +s_{1} T\left\|T_{\alpha}\left(s_{2}-s\right)-T_{\alpha}\left(s_{1}-s\right)\right\|\left(K_{1}\left(r^{\prime}+\frac{t^{\gamma}}{\Gamma(\gamma+1)}\left(r^{\prime \prime}\right)\right)+K_{2}\right)+\left(s_{2}-s_{1}\right) T \breve{M}\left(K _ { 1 } \left(r^{\prime}+\right.\right. \\
& \left.\left.\frac{t^{\gamma}}{\Gamma(\gamma+1)}\left(r^{\prime \prime}\right)\right)+K_{2}\right) \\
& \text { Also, if } t_{k}<s_{1}<s_{2} \leq t_{k+1} \text {, then we get } \\
& \left\|(\overline{\mathrm{Y}} y)\left(s_{2}\right)-(\overline{\mathrm{Y}} y)\left(s_{1}\right)\right\| \\
& \leq\left\|\sum_{i=1}^{k}\left[S_{\alpha}\left(s_{2}-t_{i}\right)+K_{\alpha}\left(s_{2}-t_{i}\right)\right]-\sum_{i=1}^{k}\left[S_{\alpha}\left(s_{1}-t_{i}\right)+K_{\alpha}\left(s_{1}-t_{i}\right)\right]\right\| \\
& \left.\times \| \int_{t_{i-1}}^{t_{i}} P_{\alpha}\left(t_{i}-s\right) h\left(s, y_{s}+\emptyset_{s}\right) d s+\int_{t_{i-1}}^{t_{i}} \int_{0}^{s} T_{\alpha}\left(t_{i}-s\right) f\left(c, y_{c}+\emptyset_{c}, I_{c}^{\gamma}(y+\emptyset)\right) d c d s\right) \| \\
& +\int_{t_{k}}^{s_{1}}\left\|P_{\alpha}\left(s_{2}-s\right)-P_{\alpha}\left(s_{1}-s\right)\right\|\left\|h\left(s, y_{s}+\emptyset_{s}\right)\right\| d s+\int_{s_{1}}^{s_{2}}\left\|P_{\alpha}\left(s_{2}-s\right)\right\|\left\|h\left(s, y_{s}+\emptyset_{s}\right)\right\| d s \\
& +\int_{t_{k}}^{s_{1}} \int_{0}^{s}\left\|T_{\alpha}\left(s_{2}-s\right)-T_{\alpha}\left(s_{1}-s\right)\right\|\left\|f\left(c, y_{c}+\emptyset_{c}, I_{c}^{\gamma}(y+\emptyset)\right)\right\| d c d s \\
& +\int_{s_{1}}^{s_{2}} \int_{0}^{s}\left\|T_{\alpha}\left(s_{2}-s\right)\right\|\left\|f\left(c, y_{c}+\emptyset_{c}, I_{c}^{\gamma}(y+\emptyset)\right)\right\| d c d s \\
& \leq \sum_{i=1}^{k}\left\|S_{\alpha}\left(s_{2}-t_{i}\right)-S_{\alpha}\left(s_{1}-t_{i}\right)\right\|+\sum_{i=1}^{k}\left\|K_{\alpha}\left(s_{2}-t_{i}\right)-K_{\alpha}\left(s_{1}-t_{i}\right)\right\| \\
& \times\left(\left(t_{i}-t_{i-1}\right) \breve{M}\left(L_{1}\left(r^{\prime}\right)+L_{2}\right)+\left(t_{i}-t_{i-1}\right) \breve{M} T\left(K_{1}\left(r^{\prime}+\frac{t^{\gamma}}{\Gamma(\gamma+1)}\left(r^{\prime}\right)\right)+K_{2}\right)\right) \\
& +\left(s_{1}-t_{k}\right)\left\|P_{\alpha}\left(s_{2}-s\right)-P_{\alpha}\left(s_{1}-s\right)\right\|\left(L_{1}\left(r^{\prime}\right)+L_{2}\right)+\left(s_{2}-s_{1}\right) \breve{M}\left(L_{1}\left(r^{\prime}\right)+L_{2}\right) \\
& +\left(s_{1}-t_{k}\right) T\left\|T_{\alpha}\left(s_{2}-s\right)-T_{\alpha}\left(s_{1}-s\right)\right\|\left(K_{1}\left(r^{\prime}+\frac{t^{\gamma}}{\Gamma(\gamma+1)}\left(r^{\prime \prime}\right)\right)+K_{2}\right)+\left(s_{2}-s_{1}\right) \breve{M} T\left(K _ { 1 } \left(r^{\prime}+\right.\right. \\
& \left.\left.\frac{t^{\gamma}}{\Gamma(\gamma+1)}\left(r^{\prime \prime}\right)\right)+K_{2}\right)
\end{aligned}
$$

From Assumption $\left(\mathrm{A}_{1}\right)$, the compactness of $S_{\alpha}(t), K_{\alpha}(t), P_{\alpha}(t)$ and $T_{\alpha}(t)$ for $t>0$ satisfy the continuity in the uniform operator topology. Then the right-hand sides of (3.10) and (3.11) tend to zero as $s_{2} \rightarrow s_{1}$. Therefore, the family $\left\{(\bar{Y} y)(t): y \in E_{r}\right\}$ satisfies the equicontinuous functions. Since the proof of equicontinuities to the case $s_{1}<s_{2}<0$ and $s_{1}<0<s_{2}$ is simple, then the proof is omitted.

\section{Lemma (3.8)}

If the assumptions $\left(\mathrm{A}_{1}\right)-\left(\mathrm{A}_{3}\right)$ hold, then the $W(t)=\left\{(\overline{\mathrm{Y}} y)(t): y \in E_{r}\right\}$ is relatively compact for any $t \in J \backslash\left\{t_{1}, \ldots, t_{m}\right\}$, where the operator $\overline{\mathrm{Y}}$ on $E_{r}$ is defined in (3.9). 


\section{Proof}

Let $t \in\left(0, t_{1}\right]$ be a fixed and $\varepsilon$ be a real number, implies $0<\varepsilon<t_{1}$. For $\in E_{r}$, we define $W_{\varepsilon}(t)=\left\{\left(\bar{\Upsilon}_{\varepsilon} y\right)(t): y \in E_{r}\right\}$ as follows:

$$
\begin{aligned}
\left(\bar{\Upsilon}_{\varepsilon} y\right)(t)= & \int_{0}^{t-\varepsilon} P_{\alpha}(t-s) h\left(s, y_{s}+\emptyset_{s}\right) d s+\int_{0}^{t-\varepsilon} \int_{0}^{s} T_{\alpha}(t-s) f\left(c, y_{c}+\emptyset_{c}, I_{c}^{\gamma}(y+\emptyset)\right) d c d s \\
= & P_{\alpha}(\varepsilon) \int_{0}^{t-\varepsilon} P_{\alpha}(t-s-\varepsilon) h\left(s, y_{s}+\emptyset_{s}\right) d s \\
& +T_{\alpha}(\varepsilon) \int_{0}^{t-\varepsilon} \int_{0}^{s} T_{\alpha}(t-s-\varepsilon) f\left(c, y_{c}+\emptyset_{c}, I_{c}^{\gamma}(y+\emptyset)\right) d c d s
\end{aligned}
$$

Also, for $t \in\left(t_{k}, t_{k+1}\right]$ to be a fixed and $\varepsilon$ be a real number, implies $t_{k}<\varepsilon<t_{k+1}$, we define the subset $W_{\varepsilon}(t)=\left\{\left(\bar{Y}_{\varepsilon} y\right)(t): y \in E_{r}\right\}$

$$
\begin{aligned}
\left(\bar{\Upsilon}_{\varepsilon} y\right)(t)= & \sum_{i=1}^{k}\left[S_{\alpha}\left(t-t_{i}\right)+K_{\alpha}\left(t-t_{i}\right)\right]\left(\int_{t_{i-1}}^{t_{i}} P_{\alpha}\left(t_{i}-s\right) h\left(s, y_{s}+\emptyset_{s}\right) d s\right. \\
& \left.+\int_{t_{i-1}}^{t_{i}} \int_{0}^{s} T_{\alpha}\left(t_{i}-s\right) f\left(c, y_{c}+\emptyset_{c}, I_{c}^{\gamma}(y+\emptyset)\right) d c d s\right)+P_{\alpha}(\varepsilon) \int_{t_{k}}^{t-\varepsilon} P_{\alpha}(t-s- \\
\varepsilon) h\left(s, y_{s}+\right. & \left.\emptyset_{s}\right) d s \\
& +T_{\alpha}(\varepsilon) \int_{t_{k}}^{t-\varepsilon} \int_{0}^{s} T_{\alpha}(t-s-\varepsilon) f\left(c, y_{c}+\emptyset_{c}, I_{c}^{\gamma}(y+\emptyset)\right) d c d s
\end{aligned}
$$

From assumption $\left(\mathrm{A}_{1}\right)-\left(\mathrm{A}_{3}\right)$, we have $S_{\alpha}(t), K_{\alpha}(t), P_{\alpha}(t)$ and $T_{\alpha}(t)$ are compact and the right-hand side of (3.12) and (3.13) are bounded, then the set $W_{\varepsilon}(t)=\left\{\left(\bar{\Upsilon}_{\varepsilon} y\right)(t): y \in E_{r}\right\}$ is relatively compact in $X$, for every $\varepsilon, t_{k}<\varepsilon<t_{k+1}$.

Moreover, for $y \in E_{r}, t \in\left(0, t_{1}\right]$, we have

$\left\|(\bar{\Upsilon} y)(t)-\left(\bar{\Upsilon}_{\varepsilon} y\right)(t)\right\| \leq \varepsilon\left\|P_{\alpha}(t-s)\right\|\left\|h\left(s, y_{s}+\emptyset_{s}\right)\right\|+\varepsilon\left\|T_{\alpha}(t-s)\right\|\left\|f\left(c, y_{c}+\emptyset_{c}, I_{c}^{\gamma}(y+\emptyset)\right)\right\|$

$$
\leq \varepsilon \breve{M}\left[\left(L_{1} r^{\prime}+L_{2}\right)+\left(K_{1}\left(r^{\prime}+\frac{t^{\gamma}}{\Gamma(\gamma+1)}\left(r^{\prime \prime}\right)\right)+K_{2}\right)\right]
$$

and for $t \in\left(t_{k}, t_{k+1}\right]$, we get

$$
\begin{aligned}
\|(\bar{\Upsilon} y)(t)-\left(\bar{\Upsilon}_{\varepsilon} y\right) & (t)\|\leq\| \sum_{i=1}^{k}\left[S_{\alpha}\left(t-t_{i}\right)+K_{\alpha}\left(t-t_{i}\right)\right]-\sum_{i=1}^{k}\left[S_{\alpha}\left(t-t_{i}-\varepsilon\right)+K_{\alpha}\left(t-t_{i}-\varepsilon\right)\right] \| \\
& \times\left[\left(t_{i}-t_{i-1}\right)\left\|P_{\alpha}\left(t_{i}-s\right)\right\|\left\|h\left(s, y_{s}+\emptyset_{s}\right)\right\|+\left(t_{i}-t_{i-1}\right) T\left\|T_{\alpha}\left(t_{i}-s\right)\right\| \| f\left(c, y_{c}+\right.\right. \\
\emptyset_{c}, I_{c}^{\gamma}(y+ & \varnothing)) \|] \\
& +\varepsilon\left\|P_{\alpha}(t-s)\right\|\left\|h\left(s, y_{s}+\emptyset_{s}\right)\right\|+\varepsilon T\left\|T_{\alpha}(t-s)\right\|\left\|f\left(c, y_{c}+\emptyset_{c}, I_{c}^{\gamma}(y+\emptyset)\right)\right\| \\
\leq & \left\|\sum_{i=1}^{k}\left[S_{\alpha}\left(t-t_{i}\right)+K_{\alpha}\left(t-t_{i}\right)\right]-\sum_{i=1}^{k}\left[S_{\alpha}\left(t-t_{i}-\varepsilon\right)+K_{\alpha}\left(t-t_{i}-\varepsilon\right)\right]\right\| \\
& \times\left[\left(t_{i}-t_{i-1}\right) \bar{M}\left(L_{1}\left(r^{\prime}\right)+L_{2}\right)+\left(t_{i}-t_{i-1}\right) T \bar{M}\left(K_{1}\left(r^{\prime}+\frac{t^{\gamma}}{\Gamma(\gamma+1)}\left(r^{\prime \prime}\right)\right)+K_{2}\right)\right] \\
& +\varepsilon \bar{M}\left(L_{1}\left(r^{\prime}\right)+L_{2}\right)+\varepsilon T \breve{M}\left(K_{1}\left(r^{\prime}+\frac{t^{\gamma}}{\Gamma(\gamma+1)}\left(r^{\prime \prime}\right)\right)+K_{2}\right)
\end{aligned}
$$

Therefore, as $\varepsilon \rightarrow 0$, there are relatively compact sets arbitrarily closed to the set $W(t)=$ $\left\{(\bar{\Upsilon} y)(t): y \in E_{r}\right\}$ for each $\in J \backslash\left\{t_{1}, \ldots, t_{m}\right\}$. Hence, the set $W(t)=\left\{(\bar{\Upsilon} y)(t): y \in E_{r}\right\}, t \in J \backslash$ $\left\{t_{1}, \ldots, t_{m}\right\}$ is relatively compact in $\mathrm{X}$.

\section{Theorem (3.1)}

If the assumptions $\left(\mathrm{A}_{1}\right)-\left(\mathrm{A}_{7}\right)$ hold, then the impulsive abstract Cauchy problem (1.2) has at least one mild solution $z$ that belongs to $E_{r}$.

\section{Proof}

It suffices to prove that the operator $\Psi$ defined as follows has a fixed point $z(\cdot)$ 


$$
\begin{aligned}
& (\Psi \mathrm{z})(t, x)
\end{aligned}
$$

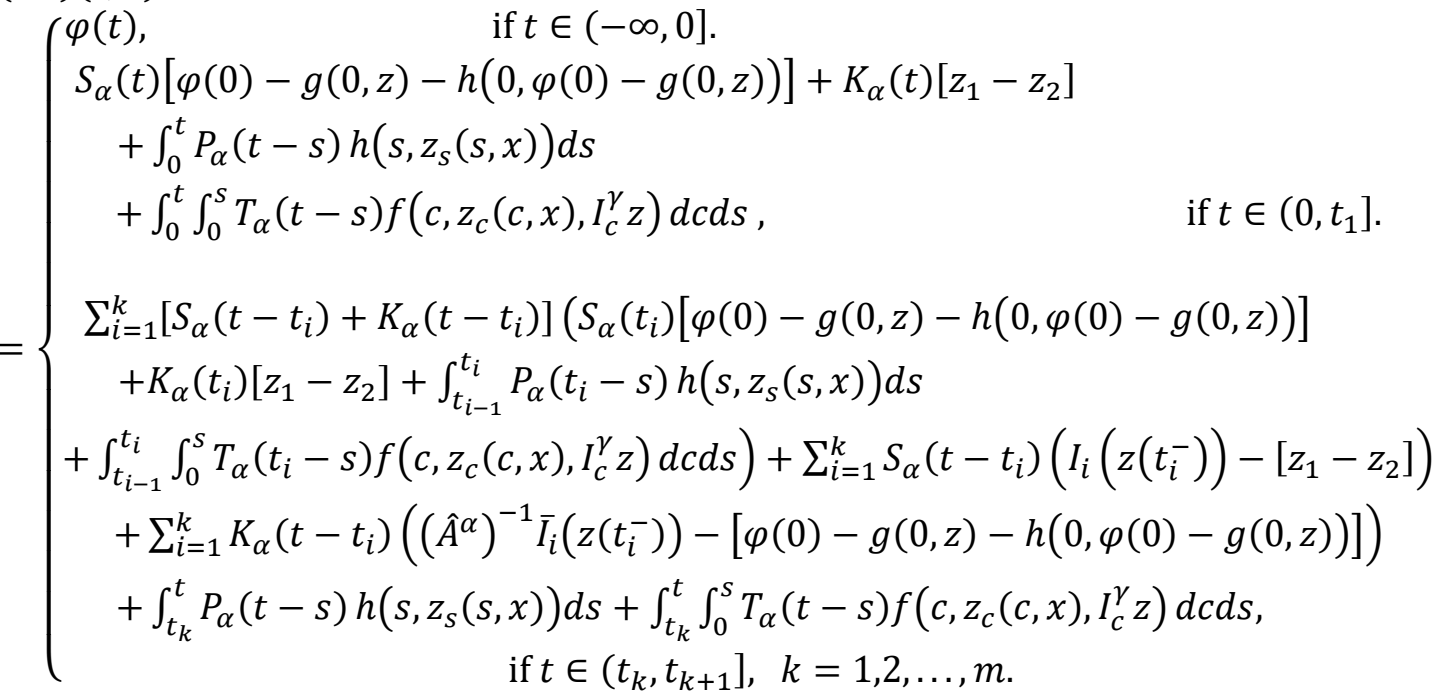

We consider the operators $Y$ and $\bar{Y}$ on $E_{r}$ defined as follows:

$(\Upsilon y)(t)$

$$
=\left\{\begin{array}{c}
0, \text { if } t \in(-\infty, 0] \\
S_{\alpha}(t)[\varphi(0)-g(0, y+\emptyset)-h(0, \varphi(0)-g(0, y+\emptyset))]+K_{\alpha}(t)\left[z_{1}-z_{2}\right] \quad \text { if } t \in\left(0, t_{1}\right] \\
\sum_{i=1}^{k}\left[S_{\alpha}\left(t-t_{i}\right)+K_{\alpha}\left(t-t_{i}\right)\right]\left(S_{\alpha}\left(t_{i}\right)[\varphi(0)-g(0, y+\emptyset)-h(0, \varphi(0)-g(y+\emptyset))]\right. \\
\left.+K_{\alpha}\left(t_{i}\right)\left[z_{1}-z_{2}\right]\right)+\sum_{i=1}^{k} S_{\alpha}\left(t-t_{i}\right)\left(I_{i}\left(y\left(t_{i}^{-}\right)+\emptyset\left(t_{i}^{-}\right)\right)-\left[z_{1}-z_{2}\right]\right) \\
+\sum_{i=1}^{k} K_{\alpha}\left(t-t_{i}\right)\left(\left(\hat{A}^{\alpha}\right)^{-1} \bar{I}_{i}\left(y\left(t_{i}^{-}\right)+\emptyset\left(t_{i}^{-}\right)\right)-[\varphi(0)-g(0, y+\emptyset)-h(0, \varphi(0)-g(0 . y+\emptyset))]\right) \\
\quad, \text { if } t \in\left(t_{k}, t_{k+1}\right], k=1,2, \ldots, m
\end{array}\right.
$$

$(\bar{\Upsilon} y)(t)$

$$
=\left\{\begin{aligned}
0, & \text { if } t \in(-\infty, 0] \\
\int_{0}^{t} & P_{\alpha}(t-s) h\left(s, y_{s}+\emptyset_{s}\right) d s+\int_{0}^{t} \int_{0}^{s} T_{\alpha}(t-s) f\left(c, y_{c}+\emptyset_{c}, I_{c}^{\gamma}(y+\emptyset)\right) d c d s, \text { if } t \in\left(0, t_{1}\right] \\
& \sum_{i=1}^{k}\left[S_{\alpha}\left(t-t_{i}\right)+K_{\alpha}\left(t-t_{i}\right)\right]\left(\int_{t_{i-1}}^{t_{i}} P_{\alpha}\left(t_{i}-s\right) h\left(s, y_{s}+\emptyset_{s}\right) d s\right. \\
& \left.+\int_{t_{i-1}}^{t_{i}} \int_{0}^{s} T_{\alpha}\left(t_{i}-s\right) f\left(c, y_{c}+\emptyset_{c}, I_{c}^{\gamma}(y+\emptyset)\right) d c d s\right)+\int_{t_{k}}^{t} P_{\alpha}(t-s) h\left(s, y_{s}+\emptyset_{s}\right) d s \\
& +\int_{t_{k}}^{t} \int_{0}^{s} T_{\alpha}(t-s) f\left(c, y_{c}+\emptyset_{c}, I_{c}^{\gamma}(y+\emptyset)\right) d c d s, \quad \text { if } t \in\left(t_{k}, t_{k+1}\right], k=1,2, \ldots, m
\end{aligned}\right.
$$

Now, to find the mild solution of impulsive abstract Cauchy problem (1.2), we need to prove that $\Upsilon+\bar{\Upsilon}$ has a fixed point on $E_{r}$. For any,$v \in E_{r}$, we have

$$
\begin{aligned}
\|(\Upsilon u)(t)+ & (\bar{Y} v)(t) \| \leq \bar{M}[\|\varphi(0)\|+\|g(0, u+\emptyset)\|+\|h(0, \varphi(0)-g(0, u+\emptyset))\|] \\
& +\breve{M}\left\|z_{1}-z_{2}\right\|+\breve{M} t_{1}\left(L_{1}\left\|v_{s}(s)+\emptyset_{s}(s)\right\|_{E_{v}}+L_{2}\right) \\
& +\breve{M} t_{1} T\left(K_{1}\left(\left\|v_{c}(s)+\emptyset_{c}(s)\right\|_{E_{v}}+\frac{t^{\gamma}}{\Gamma(\gamma+1)} \sup _{0<s \leq t_{1}}\|(v(s)+\emptyset(s))\|_{E_{v}}\right)+K_{2}\right) \\
\leq & \breve{M}[\|\varphi(0)\|+\|g(0, u+\emptyset)\|+\|h(0, \varphi(0)-g(0, u+\emptyset))\|]+\breve{M}\left\|z_{1}-z_{2}\right\| \\
& +\breve{M} t_{1}\left(L_{1} r^{\prime}+L_{2}\right)+\breve{M} t_{1} T\left(K_{1}\left(r^{\prime}+\frac{t^{\gamma}}{\Gamma(\gamma+1)} r^{\prime}\right)+K_{2}\right) \leq N \leq r, \quad \text { for } t \in\left(0, t_{1}\right]
\end{aligned}
$$

and

$\|(\Upsilon u)(t)+(\bar{\Upsilon} v)(t)\|$ 


$$
\begin{aligned}
\leq & \sum_{i=1}^{k}\left[\left\|S_{\alpha}\left(t-t_{i}\right)\right\|+\left\|K_{\alpha}\left(t-t_{i}\right)\right\|\right]\left(\left\|S_{\alpha}\left(t_{i}\right)\right\| \| \varphi(0)-g(0, u+\emptyset)-h(0, \varphi(0)-\right. \\
g(0, u+ & \emptyset)) \| \\
& \left.+\left\|K_{\alpha}\left(t_{i}\right)\right\|\left\|z_{1}-z_{2}\right\|\right)+\sum_{i=1}^{k}\left\|S_{\alpha}\left(t-t_{i}\right)\right\|\left(a_{i}\left\|u\left(t_{i}^{-}\right)+\emptyset\left(t_{i}^{-}\right)\right\|+b_{i}-\left\|z_{1}-z_{2}\right\|\right) \\
& +\sum_{i=1}^{k}\left\|K_{\alpha}\left(t-t_{i}\right)\right\|\left(c_{i}\left\|u\left(t_{i}^{-}\right)+\emptyset\left(t_{i}^{-}\right)\right\|+d_{i}+\| \varphi(0)-g(0, u+\emptyset)-h(0, \varphi(0)-\right. \\
g(0, u+ & \emptyset)) \|) \\
& +\sum_{i=1}^{k}\left[\left\|S_{\alpha}\left(t-t_{i}\right)\right\|+\left\|K_{\alpha}\left(t-t_{i}\right)\right\|\right]\left(\int_{t_{i-1}}^{t_{i}}\left\|P_{\alpha}\left(t_{i}-s\right)\right\|\left(L_{1}\left\|v_{s}(s)+\emptyset_{s}(s)\right\|+L_{2}\right) d s\right. \\
& \left.+\int_{t_{i-1}}^{t_{i}} \int_{0}^{s}\left\|T_{\alpha}\left(t_{i}-s\right)\right\|\left(K_{1}\left(\left\|v_{c}+\emptyset_{c}\right\|+\left\|I_{c}^{\gamma}(v+\emptyset)\right\|\right)+K_{2}\right) d c d s\right) \\
& +\int_{t_{k}}^{t}\left\|P_{\alpha}(t-s)\right\|\left(L_{1}\left\|v_{s}(s)+\emptyset_{s}(s)\right\|+L_{2}\right) d s \\
& +\int_{t_{k}}^{t} \int_{0}^{s}\left\|T_{\alpha}(t-s)\right\|\left(K_{1}\left(\left\|v_{c}+\emptyset_{c}\right\|+\left\|I_{c}^{\gamma}(v+\emptyset)\right\|\right)+K_{2}\right) d c d s \\
\leq & \sum_{i=1}^{k} 2 \breve{M}\left(\breve{M}\|\varphi(0)-g(0, u+\emptyset)-h(0, \varphi(0)-g(0, u+\emptyset))\|+\breve{M}\left\|z_{1}-z_{2}\right\|\right) \\
& +\sum_{i=1}^{k} \bar{M}\left(a_{i}\left(r^{\prime \prime}\right)+b_{i}-\left\|z_{1}-z_{2}\right\|\right) \\
& +\sum_{i=1}^{k} \bar{M}\left(c_{i}\left(r^{\prime \prime}\right)+d_{i}+\|\varphi(0)-g(0, u+\emptyset)-h(0, \varphi(0)-g(0, u+\emptyset))\|\right) \\
& +\sum_{i=1}^{k} 2 \breve{M}\left(\left(t_{i}-t_{i-1}\right) \breve{M}\left(L_{1}\left(r^{\prime}\right)+L_{2}\right)+\left(t_{i}-t_{i-1}\right) \bar{M} T\left(K_{1}\left(r^{\prime}+\frac{t^{\gamma}}{\Gamma(\gamma+1)}\left(r^{\prime}\right)\right)+K_{2}\right)\right. \\
& +\breve{M} T\left(L_{1}\left(r^{\prime}\right)+L_{2}\right)+\breve{M} T^{2}\left(K_{1}\left(r^{\prime}+\frac{t^{\gamma}}{\Gamma(\gamma+1)}\left(r^{\prime \prime}\right)\right)+K_{2}\right) \leq N \leq r .
\end{aligned}
$$

For $t \in\left(t_{k}, t_{k+1}\right]$, then we can get $\rho_{1}, \rho_{2}>0$, such that

$$
\|(\Upsilon u)(t)+(\bar{\Upsilon} v)(t)\| \leq\left\{\begin{array}{l}
\rho_{1}, t \in\left[0, t_{1}\right] \\
\rho_{2}, t \in\left[t_{k}, t_{k+1}\right], k=1,2, \ldots, m
\end{array} \leq N \leq r .\right.
$$

Which means that $\Upsilon u+\bar{\Upsilon} v$ is bounded and thus verified the condition (1) in Lemma (2.2).

Now, we show that $\bar{\Upsilon}$ is compact. For this we start proving that $\left\{(\bar{\Upsilon} y)(t): y \in E_{r}\right\}$ is relatively compact, for $t \in\left[0, t_{1}\right]$, we have

$$
\begin{aligned}
\|(\bar{\Upsilon} y)(t)\| & \leq\left\|\int_{0}^{t_{1}} P_{\alpha}(t-s) h\left(s, y_{s}+\emptyset_{s}\right) d s\right\|+\left\|\int_{0}^{t_{1}} \int_{0}^{s} T_{\alpha}(t-s) f\left(c, y_{c}+\emptyset_{c}, I_{c}^{\gamma}(y+\emptyset)\right) d c d s\right\| \\
& \leq \breve{M} t_{1}\left(L_{1}\left(\|\varphi\|_{E_{v}}+l r+\bar{M}\|[\varphi(0)-g(0, u+\emptyset)]\|\right)+L_{2}\right) \\
& +\breve{M} t_{1} T\left(K _ { 1 } \left(\|\varphi\|_{E_{v}}+l r+\breve{M}\|[\varphi(0)-g(0, u+\emptyset)]\|+\frac{t^{\gamma}}{\Gamma(\gamma+1)}(r+\breve{M} \| \varphi(0)-\right.\right. \\
\left.g(0, u+\emptyset) \|))+K_{2}\right) & \\
& \leq \breve{M} t_{1}\left[\left(L_{1}\left(r^{\prime}\right)+L_{2}\right)+T\left(K_{1}\left(r^{\prime}+\frac{t^{\gamma}}{\Gamma(\gamma+1)}\left(r^{\prime}\right)\right)+K_{2}\right)\right]
\end{aligned}
$$

and for $t \in\left(t_{k}, t_{k+1}\right]$, we get

$$
\begin{aligned}
\|(\bar{\Upsilon} y)(t)\|= & \| \sum_{i=1}^{k}\left[S_{\alpha}\left(t-t_{i}\right)+K_{\alpha}\left(t-t_{i}\right)\right]\left(\int_{t_{i-1}}^{t_{i}} P_{\alpha}\left(t_{i}-s\right) h\left(s, y_{s}+\emptyset_{s}\right) d s\right. \\
& \left.+\int_{t_{i-1}}^{t_{i}} \int_{0}^{s} T_{\alpha}\left(t_{i}-s\right) f\left(c, y_{c}+\emptyset_{c}, I_{c}^{\gamma}(y+\emptyset)\right) d c d s\right)+\int_{t_{k}}^{t} P_{\alpha}(t-s) h\left(s, y_{s}+\emptyset_{s}\right) d s \\
& +\int_{t_{k}}^{t} \int_{0}^{s} T_{\alpha}(t-s) f\left(c, y_{c}+\emptyset_{c}, I_{c}^{\gamma}(y+\emptyset)\right) d c d s \| \\
\leq & \sum_{i=1}^{k} 2 \breve{M}\left(\left(t_{i}-t_{i-1}\right) \breve{M}\left(\left(L_{1} r^{\prime}+L_{2}\right)\right)+\left(t_{i}-t_{i-1}\right) \breve{M} T\left(\left(K_{1}\left(r^{\prime}+\frac{t^{\gamma}}{\Gamma(\gamma+1)}\left(r^{\prime \prime}\right)\right)+K_{2}\right)\right)\right) \\
+ & \breve{M} T\left(\left(L_{1}\left(r^{\prime}\right)+L_{2}\right)\right)+\breve{M} T^{2}\left(\left(K_{1}\left(r^{\prime}+\frac{t^{\gamma}}{\Gamma(\gamma+1)}\left(r^{\prime \prime}\right)\right)+K_{2}\right)\right) .
\end{aligned}
$$

Then the set $\left\{(\bar{Y} y)(t): y \in E_{r}\right\}$ is uniformly bounded. Therefore, from lemma (3.7), we have that the $\bar{\Upsilon}\left(E_{r}\right)$ is equicontinuous, and from lemma (3.8), we have that the $W(t)=\left\{(\bar{\Upsilon} y)(t): y \in E_{r}\right\}$ is relatively compact for any $t \in J \backslash\left\{t_{1}, \ldots, t_{m}\right\}$. Then by the Arzela-Ascoli theorem, we get that the closure of $\left\{(\bar{\Upsilon} y)(t): y \in E_{r}\right\}$ is compact . Next, we shall prove that $\bar{\Upsilon}$ is continuous:

Let $\left\{y_{n}\right\}$ be a sequence in $E_{r}$ and $y_{n} \rightarrow y$ for $\in E_{v}$. From assumptions $\left(\mathrm{A}_{2}\right)-\left(\mathrm{A}_{3}\right)$, we get $f$ and $h$ are continuous, i.e. for all $\varepsilon>0$ there is a positive integer $N$ such that for $n>N$

$$
\left\|f\left(c, y_{c_{n}}+\emptyset_{c_{n}}, I_{c}^{\gamma}\left(y_{n}+\emptyset_{n}\right)\right)-f\left(c, y_{c}+\emptyset_{c}, I_{c}^{\gamma}(y+\emptyset)\right)\right\| \leq \varepsilon \quad \text { and } \quad \| h\left(s, y_{s_{n}}+\emptyset_{s_{n}}\right)-
$$
$h\left(s, y_{s}+\emptyset_{s}\right) \| \leq \varepsilon$.

Now, for $\in\left(0, t_{1}\right]$, we get

$\left\|\left(\bar{\Upsilon} y_{n}\right)(t)-(\bar{\Upsilon} y)(t)\right\| \leq \int_{0}^{t_{1}}\left\|P_{\alpha}(t-s)\right\|\left\|h\left(s, y_{s_{n}}+\emptyset_{s_{n}}\right)-h\left(s, y_{s}+\emptyset_{s}\right)\right\| d s$ 


$$
+\int_{0}^{t_{1}} \int_{0}^{s}\left\|T_{\alpha}(t-s)\right\| \| f\left(c, y_{c_{n}}+\emptyset_{c_{n}}, I_{c}^{\gamma}\left(y_{n}+\emptyset_{n}\right)\right)-f\left(c, y_{c}+\right.
$$

$\left.\emptyset_{c}, I_{c}^{\gamma}(y+\emptyset)\right) \| d c d s$

$$
\begin{aligned}
& \leq t_{1} \breve{M}\left\|h\left(s, y_{s_{n}}+\emptyset_{s_{n}}\right)-h\left(s, y_{s}+\emptyset_{s}\right)\right\| \\
& +t_{1} \breve{M} T\left\|f\left(c, y_{c_{n}}+\emptyset_{c_{n}}, I_{c}^{\gamma}\left(y_{n}+\emptyset_{n}\right)\right)-f\left(c, y_{c}+\emptyset_{c}, I_{c}^{\gamma}(y+\emptyset)\right)\right\| \\
& \leq t_{1} \breve{M} \varepsilon(1+T)
\end{aligned}
$$

Moreover, for $\in\left(t_{k}, t_{k+1}\right]$, we have

$$
\begin{array}{ll}
\|\left(\bar{\Upsilon} y_{n}\right)(t)- & (\bar{\Upsilon} y)(t) \| \\
\leq & \sum_{i=1}^{k}\left\|S_{\alpha}\left(t-t_{i}\right)+K_{\alpha}\left(t-t_{i}\right)\right\|\left(\int_{t_{i-1}}^{t_{i}}\left\|P_{\alpha}\left(t_{i}-s\right)\right\| \| h\left(s, y_{s_{n}}+\emptyset_{s_{n}}\right)-h\left(s, y_{s}+\right.\right. \\
\left.\emptyset_{s}\right) \| d s \quad & +\int_{t_{i-1}}^{t_{i}} \int_{0}^{s}\left\|T_{\alpha}\left(t_{i}-s\right)\right\| \| f\left(c, y_{c_{n}}+\emptyset_{c_{n}}, I_{c}^{\gamma}\left(y_{n}+\emptyset_{n}\right)\right)-f\left(c, y_{c}+\emptyset_{c}, I_{c}^{\gamma}(y+\right. \\
\emptyset)) \| d c d s) & \\
& +\int_{t_{k}}^{t}\left\|P_{\alpha}(t-s)\right\|\left\|h\left(s, y_{s_{n}}+\emptyset_{s_{n}}\right)-h\left(s, y_{s}+\emptyset_{s}\right)\right\| d s \\
& +\int_{t_{k}}^{t} \int_{0}^{s}\left\|T_{\alpha}(t-s)\right\| \| f\left(c, y_{c_{n}}+\emptyset_{c_{n}}, I_{c}^{\gamma}\left(y_{n}+\emptyset_{n}\right)\right)-f\left(c, y_{c}+\emptyset_{c}, I_{c}^{\gamma}(y+\right. \\
& \varnothing)) \| d c d s \\
\leq & \sum_{i=1}^{k} \bar{M} \varepsilon\left(t_{i}-t_{i-1}\right)(2 \breve{M}+T) \\
& +\bar{M} \varepsilon\left(t_{k}-t_{k+1}\right)(1+T)
\end{array}
$$

Hence, $\bar{\Upsilon}$ is continuous. By the analysis above, we can see that $\bar{\Upsilon}$ implies the condition (2) of lemma (2.2) which means that $\bar{Y}$ is completely continuous.

For ending the proof, we will show that $\Upsilon$ is a contraction. Let , $v \in E_{r}$, for $t \in\left(0, t_{1}\right]$, we get

$$
\begin{aligned}
\|(\Upsilon u)(t)-(\Upsilon v)(t)\| \leq & \left\|S_{\alpha}(t)\right\| \|[\varphi(0)-g(0, u+\emptyset)-h(0, \varphi(0)-g(0, u+\emptyset))] \\
& -[\varphi(0)-g(0, v+\emptyset)-h(0, \varphi(0)-g(0, v+\emptyset))] \| \\
\leq & \left\|S_{\alpha}(t)\right\|\left(N_{1}\|u-v\|+L_{1}\left(N_{1}\|u-v\|\right)\right) \\
\leq & \widetilde{M}\left(1+L_{1}\right) N_{1}\|u-v\|
\end{aligned}
$$

Therefore, for $\in\left(t_{k}, t_{k+1}\right]$, we get

$$
\begin{aligned}
& \|(\Upsilon u)(t)-(\Upsilon v)(t)\| \leq \sum_{i=1}^{k} 2 \breve{M}\left(\breve{M}\left(N_{1}\|u-v\|+L_{1}\left(N_{1}\|u-v\|\right)\right)\right) \\
& \quad+\sum_{i=1}^{k} \breve{M} d_{i}\left\|u\left(t_{i}^{-}\right)-v\left(t_{i}^{-}\right)\right\|+\sum_{i=1}^{k} \breve{M}\left(\bar{d}_{i}\left\|u\left(t_{i}^{-}\right)-v\left(t_{i}^{-}\right)\right\|+N_{1}\|u-v\|+\right. \\
& \quad \leq\left[\sum_{i=1}^{k} 2 \breve{M} N_{1}\left(\breve{M}\left(1+L_{1}\right)\right)+\sum_{i=1}^{k} \breve{M} d_{i}+\sum_{i=1}^{k} \breve{M}\left(\bar{d}_{i}+N_{1}+L_{1}\left(N_{1}\right)\right)\right]\|u-v\|
\end{aligned}
$$

Since $\breve{M}\left(1+L_{1}\right) N_{1}<1$, and

$$
\left[\sum_{i=1}^{k} 2 \breve{M} N_{1}\left(\breve{M}\left(1+L_{1}\right)\right)+\sum_{i=1}^{k} \breve{M} d_{i}+\sum_{i=1}^{k} \breve{M}\left(\bar{d}_{i}+N_{1}+L_{1}\left(N_{1}\right)\right)\right]<1 \quad, \quad \text { for } \quad k=
$$

$1,2, \ldots, m$.

Then $\Upsilon$ is contraction. Therefore, the three conditions of lemma (2.2) are satisfied. Therefore, for this the operator $\Upsilon+\bar{\Upsilon}$ has a fixed point in $E_{r}$. Then the impulsive abstract Cauchy problem (1.2) has a mild solution on $\mathrm{J}$.

\section{Conclusions:}

We conclude that the solvability of impulsive nonlinear fractional order partial differential equations with nonlocal conditions needed to define an approach to use the fractional Laplace transforms which make important roles for computing the formula of the semigroup family operators. Hence, we thought that it is an important and basic issue for solvability. The assumptions presented in this work are needed to prove that our problem, with the specific main results, was never assumed before for solving other problems. We used this approach to simulate the theoretical approach and gain solvability results of impulsive nonlinear fractional order partial differential equations with nonlocal conditions by transforming it to an impulsive abstract Cauchy problem. We also used the nonlinearity functional analysis as a suitable analytic tool for specific spaces and domains of operators to generalize the problem of nonlinear extensible beam equations and other problems.

\section{Reference}


1. Khan R. A. and Shah K. 2015. Existence and uniqueness of solutions to fractional order multipoint boundary value problems, Applied Analysis, 19: 515-526.

2. Agarwal R. P., Ntouyas S. K., Ahmad B. and Alhothuali M. S. 2013. Existence of solutions for integro-differential equations of fractional order with nonlocal three-point fractional boundary conditions, Advances in Difference Equations, (1): 128.

3. Chen S. and Liu Y. 2014. Solvability of boundary value problems for fractional order elastic beam equations, Advances in Difference Equations, 2014: 204.

4. Hu Z., Liu W.and Liu J. 2014. Boundary value problems for fractional differential equations, Boundary Value Problems, 2014: 176.

5. Guo L., Liu L., Wu Y. 2016. Existence of positive solutions for singular fractional differential equations with infinite-point boundary conditions, Nonlinear Analysis: Modelling and Control, 21(5): 635-650.

6. Qiao Y. and Zhou Z. 2017. Existence of positive solutions of singular fractional differential equations with infinite-point boundary conditions, Advances in Difference Equations, 2017: 8.

7. Lakoud A. G. and Khaldi R. 2017. Positive Solutions for Multi-order Nonlinear Fractional Systems, International Journal of Analysis and Applications. 15(1): 18-22.

8. Lv T., Pang H. and Cao L. 2018. Existence Results for Fractional Differential Equations with Multistrip Riemann-Stieltjes Integral Boundary Conditions, Hindawi Discrete Dynamics in Nature and Society, Volume, Article ID 2352789, 8 pages.

9. Bazgir H. and Ghazanfari B. 2018. Existence of Solutions for Fractional Integro-Differential Equations with Non-Local Boundary Conditions, Math. Comput. Appl. 23, 36.

10. Shu X. B. and Wang Q. 2012.The existence and uniqueness of mild solutions for fractional differential equations with nonlocal conditions of order $1<\alpha<2$, Computers and Mathematics with Applications, 64: 2100-2110.

11. Wang J., Fe ckan M., and Zhou Y. 2011. On the new concept of solutions and existence results for impulsive fractional evolution equations, Dynamics of PDE, 8(4): 345-361.

12. Chalishajar D. N., Karthikeyan K., Trujillo J. J. 2012. Existence of Mild Solutions for Fractional Impulsive Semilinear Integro-differential Equations in Banach Spaces, 19(4): 45-56.

13. Ahmad B., Malar K., and Karthikeyan K. 2013. A study of nonlocal problems of impulsive integrodifferential equations with measure of noncompactness, Advances in Difference Equations, 2013: 205.

14. Kumar P., Pandey D. N., Bahuguna D. 2014. On a new class of abstract impulsive functional differential equations of fractional order, J. Nonlinear Sci. Appl. 7: 102-114.

15. Fu X., Liu X. and Lu B. 2015. On a new class of impulsive fractional evolution equations, Advances in Difference Equations, 2015: 227.

16. Li B. and Gou H. 2017. Existence of solutions for impulsive fractional evolution equations with periodic boundary condition, Advances in Difference Equations, 2017: 236.

17. Hial K., Guida K., Ibnelazyz L., and Oukessou M. 2018. Existence results for an impulsive fractional integro-differential equations with a non-compact semigroup, Journal of Universal Mathematics, 1(3): 293-311.

18. Anguraj A. and Kanjanadevi S. 2017. Non-Instantaneous Impulsive Fractional Neutral Differential Equations with State-Dependent Delay, Progr. Fract. Differ. Appl. 3(3): 207-218.

19. Fang H. and Song M. 2018. Existence results for fractional order impulsive functional differential equations with multiple delays, Advances in Difference Equations, 2018: 139.

20. Janaki M., Elsayed E., Kanagarajan K. 2018. Katugampola-type fractional differential equations with delay and impulses, J. Math. Theor. Phy. 1(3): 73-77.

21. Podlubny I. 1999. Fractional Differential Equations, Academic Press, San Diego. California, USA.

22. Chang Y.K. 2007. Controllability of impulsive functional differential systems with infinite delay in Banach spaces, Chaos, Solitons and Fractals, 33: 1601-1609.

23. Pazy, A. 1983. Semigroup of Linear Operator and Applications to Partial Differential Equations, Springer-Verlag, New York. 